\title{
Flow and Heat Transfer of Two Immiscible Fluids in the Presence of Uniform Inclined Magnetic Field
}

\author{
Dragiša Nikodijević, Živojin Stamenković, Dragica Milenković, \\ Bratislav Blagojević, and Jelena Nikodijevic
}

Faculty of Mechanical Engineering, University of Niš, Nis, Serbia

Correspondence should be addressed to Živojin Stamenković, zikas@masfak.ni.ac.rs

Received 17 December 2010; Revised 12 April 2011; Accepted 23 May 2011

Academic Editor: Muhammad R. Hajj

Copyright (c) 2011 Dragiša Nikodijević et al. This is an open access article distributed under the Creative Commons Attribution License, which permits unrestricted use, distribution, and reproduction in any medium, provided the original work is properly cited.

\begin{abstract}
The magnetohydrodynamic (MHD) Couette flow of two immiscible fluids in a horizontal channel with isothermal walls in the presence of an applied electric and inclined magnetic field has been investigated in the paper. Both fluids are electrically conducting, while the channel plates are electrically insulated. The general equations that describe the discussed problem under the adopted assumptions are reduced to ordinary differential equations, and closed-form solutions are obtained in both fluid regions of the channel. Separate solutions with appropriate boundary conditions for each fluid have been obtained, and these solutions have been matched at the interface using suitable matching conditions. The analytical results for various values of the Hartmann number, the angle of magnetic field inclination, loading parameter, and the ratio of fluid heights have been presented graphically to show their effect on the flow and heat transfer characteristics.
\end{abstract}

\section{Introduction}

The flow and heat transfer of electrically conducting fluids in channels and circular pipes under the effect of a transverse magnetic field occurs in magnetohydrodynamic (MHD) generators, pumps, accelerators, and flowmeters and have applications in nuclear reactors, filtration, geothermal systems, and others.

The interest in the outer magnetic field effect on heat-physical processes appeared seventy years ago. Blum et al. [1] carried out one of the first works in the field of mass and heat transfer in the presence of a magnetic field. The flow and heat transfer of a viscous incompressible electrically conducting fluid between two infinite parallel insulating plates have been studied by many researchers [2-6] due to its important applications in the further development of MHD technology. Also convective heat transfer in channels has 
been an important research topic for the last few decades because of its applications in solar technology, safety aspects of gas cooled reactors and crystal growth in liquids, and so forth.

Yang and $\mathrm{Yu}$ [7] studied the problem of convective magnetohydrodynamic channel flow between two parallel plates subjected simultaneously to an axial temperature gradient and a pressure gradient numerically. The problem of an unsteady two-dimensional flow of a viscous incompressible and electrically conducting fluid between two parallel plates in the presence of a uniform transverse magnetic field has been analyzed by Bodosa and Borkakati [8] for the case of isothermal plates and one isothermal and other adiabatic. The MHD fully developed flow and heat transfer of an electrically conducting fluid between two parallel plates with temperature-dependent viscosity is studied in $[9,10]$. An analytical solution to the problem of steady and unsteady hydromagnetic flow of viscous incompressible electrically conducting fluid under the influence of constant and periodic pressure gradient in presence of inclined magnetic field has been obtained exactly by Ghosh [11]. Borkakati and Chakrabarty [12] investigated the unsteady free convection MHD flow between two heated vertical parallel plates in induced magnetic field. Analytical investigation of laminar heat convection in a Couette-Poiseuille flow between two parallel plates with a simultaneous pressure gradient and an axial movement of the upper plate was carried out by Aydin and Avci [13]. Recently, Singha [14] gave an analytical solution to the problem of MHD free convective flow of an electrically conducting fluid between two heated parallel plates in the presence of an induced magnetic field.

All the mentioned studies pertain to a single-fluid model. Most of the problems relating to the petroleum industry, geophysics, plasma physics, magneto-fluid dynamics, and so forth involve multifluid flow situations. Hartmann flow of a conducting fluid and a nonconducting fluid layer contained in a channel has been studied by Shail [15]. His results predicted that an increase of the order $30 \%$ can be achieved in the flow rate for suitable ratios of heights and viscosities of the two fluids. Lohrasbi and Sahai [16] studied two-phase MHD flow and heat transfer in a parallel plate channel with the fluid in one phase being conducting. These studies are expected to be useful in understanding the effect of the presence of a slag layer on heat transfer characteristics of a coal-fired MHD generator.

There have been some experimental and analytical studies on hydrodynamic aspects of the two-fluid flow reported in the recent literature. Following the ideas of Alireza and Sahai [17], Malashetty et al. [18, 19] have studied the two fluid MHD flow and heat transfer in an inclined channel, and flow in an inclined channel containing porous and fluid layer. Umavathi et al. [20, 21] have presented analytical solutions of an oscillatory Hartmann two-fluid flow and heat transfer in a horizontal channel and an unsteady two-fluid flow and heat transfer in a horizontal channel. Recently, Umavathi et al. [22] have analysed the magnetohydrodynamic Poiseuille-Couette flow and heat transfer of two immiscible fluids between inclined parallel plates.

Recent studies show that magnetohydrodynamic (MHD) flows can also be a viable option for transporting weakly conducting fluids in microscale systems, such as flow inside the microchannel networks of a lab-on-a-chip device [23, 24]. In microfluidic devices, multiple fluids may be transported through a channel for various reasons. For example, increase in mobility of a fluid may be achieved by stratification of a highly mobile fluid or mixing of two or more fluids in transit may be designed for emulsification or heat and mass transfer applications. In that regard, magnetic field-driven micropumps are in increasing demand due to their long-term reliability in generating flow, absence of moving parts, low power requirement, flow reversibility, feasibility of buffer solution manipulation, and mixing efficiency $[25,26]$. 


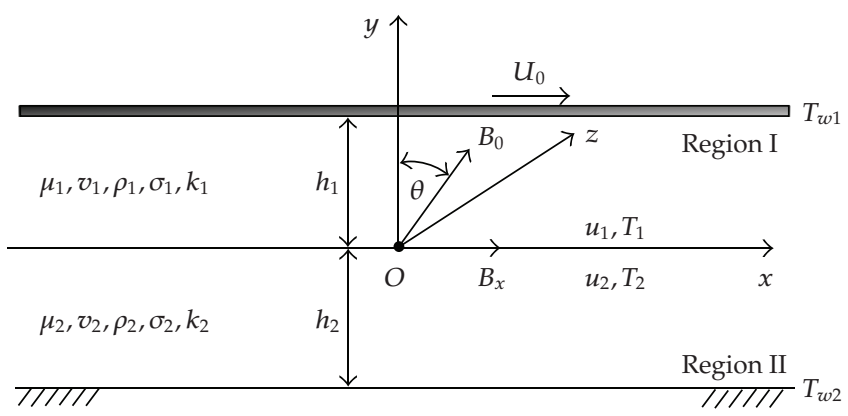

Figure 1: Physical model and coordinate system.

MHD flows inside channels can be propelled in many different ways, for example, in electromagnetohydrodynamics (EMHDs) axial flow along a channel is generated by the interaction between the magnetic field and an electric field acting normal to it. Regardless of the purpose of a multifluid EMHD flow, it is important to understand the dynamics of interfaces between the fluids and its effect on the transport characteristics of the system. Keeping in view the wide area of practical importance of multifluid flows as mentioned above, it is the objective of the present study to investigate the MHD Couette flow and heat transfer of two immiscible fluids in a parallel-plate channel in the presence of applied electric and inclined magnetic fields.

\section{Mathematical Model}

As mentioned in the introduction, the problem of the EMHD Couette two fluid flow has been considered in this paper. The fluids in the two regions have been assumed immiscible and incompressible, and the flow has been steady, one-dimensional, and fully developed. Furthermore, the two fluids have different kinematic viscosities $v_{1}$ and $v_{2}$ and densities $\rho_{1}$ and $\rho_{2}$. The transport properties of the two fluids have been taken to be constant. The analytical solutions for velocities, magnetic field, and temperature distributions have been obtained and computed for different values of the characteristic parameters. The physical model, shown in Figure 1, consists of two infinite parallel plates extending in the $x$ and $z$-direction. The upper plate moves with constant velocity $U_{0}$ in longitudinal direction. The region I $0 \leq y \leq h_{1}$ has been occupied by a fluid of viscosity $\mu_{1}$, electrical conductivity $\sigma_{1}$, thermal conductivity $k_{1}$, and specific heat capacity $c_{p 1}$, and the region II $-h_{2} \leq y \leq 0$ has been filled by a layer of different fluid of viscosity $\mu_{2}$, thermal conductivity $k_{2}$, specific heat capacity $c_{p 2}$, and electrical conductivity $\sigma_{2}$.

A uniform magnetic field of the strength $B_{0}$ has been applied in the direction making an angle $\theta$ to the vertical line and due to the fact that the fluid motion magnetic field of the strength $B_{x}$ has been induced along the lines of motion.

The fluid velocity, treating the problem as a monodimensional, and the magnetic field distributions for the case of inclined and induced magnetic field $[8,11,27,28]$ are

$$
\begin{gathered}
\mathbf{v}=(u(y), 0,0), \\
\mathbf{B}=\left(B_{x}(y)+B_{0} \sqrt{1-\lambda^{2}}, B_{0} \lambda, 0\right),
\end{gathered}
$$


where $\mathbf{B}$ is magnetic field vector and $\lambda=\cos \theta$. The upper and lower plates have been kept at the two constant temperatures $T_{w 1}$ and $T_{w 2}$, respectively, and the plates are electrically insulated. The described MHD two fluid flow problem is mathematically presented with a continuity equation:

$$
\nabla \mathbf{v}=0
$$

momentum equation:

$$
\rho\left\{\frac{\partial \mathbf{v}}{\partial t}+(\mathbf{v} \nabla) \mathbf{v}\right\}=-\nabla p+\mu \nabla^{2} \mathbf{v}+\mathbf{J} \times \mathbf{B}
$$

general magnetic induction equation:

$$
\frac{\partial \mathbf{B}}{\partial t}-\nabla \times(\mathbf{v} \times \mathbf{B})-\frac{1}{\sigma \mu_{e}} \nabla^{2} \mathbf{B}=0
$$

and an energy equation:

$$
\rho c_{p}\left(\frac{\partial T}{\partial t}+\mathbf{v} \nabla T\right)=k \nabla^{2} T+\mu \Phi+\frac{\mathbf{J}^{2}}{\sigma}
$$

where:

$$
\begin{aligned}
\Phi= & 2\left[\left(\frac{\partial u}{\partial x}\right)^{2}+\left(\frac{\partial v}{\partial y}\right)^{2}+\left(\frac{\partial w}{\partial z}\right)^{2}\right]+\left(\frac{\partial v}{\partial x}+\frac{\partial u}{\partial y}\right)^{2}+\left(\frac{\partial w}{\partial y}+\frac{\partial v}{\partial z}\right)^{2} \\
& +\left(\frac{\partial u}{\partial z}+\frac{\partial w}{\partial x}\right)^{2}-\frac{2}{3}(\nabla \mathbf{v})^{2}
\end{aligned}
$$

In previous equations and in following boundary conditions, used symbols are common for the theory of MHD flows: $t$-time, $c_{p}$-specific heat capacity, $u$-velocity in longitudinal direction, $T$-thermodynamic temperature, $\mu_{e}$-magnetic permeability and $\Phi$-dissipation function. The third term on the right hand side of (2.3) is the magnetic body force, and $\mathbf{J}$ is the current density vector due to the magnetic field and electric field defined by

$$
\mathbf{J}=\sigma(\mathbf{E}+\mathbf{v} \times \mathbf{B})
$$

where $\mathbf{E}=\left(0,0, E_{z}\right)$ is the vector of the applied electric field. 
Finally the continuity, momentum, and induction equation written in the classic quasistatic low magnetic Reynolds number approximation $[29,30]$ takes the following form:

$$
\begin{gathered}
\frac{1}{\rho} P+v \frac{d^{2} u}{d y^{2}}-\frac{\sigma}{\rho} B_{0} \lambda\left(E_{z}+u B_{0} \lambda\right)=0, \\
B_{0} \lambda \frac{d u}{d y}+\frac{1}{\sigma \mu_{e}} \frac{d^{2} B_{x}}{d y^{2}}=0, \\
\rho c_{p} u \frac{\partial T}{\partial x}=k \frac{\partial^{2} T}{\partial y^{2}}+\mu\left(\frac{\partial u}{\partial y}\right)^{2}+\sigma\left(E_{z}+u B_{0} \lambda\right)^{2},
\end{gathered}
$$

where

$$
P=-\frac{\partial p}{\partial x}
$$

The fluid and thermal boundary conditions have been unchanged by the addition of electromagnetic fields. The no-slip conditions require that the fluid velocities are equal to the plate's velocities, and boundary conditions on temperature are isothermal conditions. In addition, the fluid velocity, sheer stress, induced magnetic field, induced magnetic flux (induced currents at the interface of conductors [29]), temperature, and heat flux must be continuous across the interface $y=0$. Equations which represent these conditions are

$$
\begin{gathered}
u_{1}\left(h_{1}\right)=U_{0}, \quad u_{2}\left(-h_{2}\right)=0, \\
u_{1}(0)=u_{2}(0), \\
\mu_{1} \frac{d u_{1}}{d y}=\mu_{2} \frac{d u_{2}}{d y}, \quad y=0, \\
B_{x 1}\left(h_{1}\right)=0, \quad B_{x 2}\left(-h_{2}\right)=0, \\
B_{x 1}(0)=B_{x 2}(0), \\
\frac{1}{\mu_{e 1} \sigma_{1}} \frac{d B_{x 1}}{d y}=\frac{1}{\mu_{e 2} \sigma_{2}} \frac{d B_{x 2}}{d y} \quad \text { for } y=0, \\
T_{1}\left(h_{1}\right)=T_{w 1}, \quad T_{2}\left(-h_{2}\right)=T_{w 2}, \\
T_{1}(0)=T_{2}(0), \\
k_{1} \frac{d T_{1}}{d y}=k_{2} \frac{d T_{2}}{d y} ; \quad y=0 .
\end{gathered}
$$




\section{Velocity and Magnetic Field Distribution}

The governing equation for the velocity $u_{i}$ in regions I and II can be written as:

$$
\frac{1}{\rho_{i}} P+v_{i} \frac{d^{2} u_{i}}{d y^{2}}-\frac{\sigma_{i}}{\rho_{i}} B_{0} \lambda\left(E_{z}+u_{i} B_{0} \lambda\right)=0,
$$

where suffix $i(i=1,2)$ represents the values for regions I and II, respectively. The equation for the magnetic field induction in the regions I and II can be written as

$$
B_{0} \lambda \frac{d u_{i}}{d y}+\frac{1}{\sigma_{i} \mu_{e i}} \frac{d^{2} B_{x i}}{d y^{2}}=0
$$

It is convenient to transform (3.1) and (3.2) to a nondimensional form. The following transformations have been used:

$$
\begin{gathered}
u_{i}^{*}=\frac{u_{i}}{U_{0}}, \quad y_{i}^{*}=\frac{y}{h_{i}}, \\
\alpha=\frac{\mu_{1}}{\mu_{2}}, \quad \beta=\frac{h_{1}}{h_{2}}, \quad \gamma=\frac{\sigma_{1}}{\sigma_{2}}, \quad \delta=\frac{\mu_{e 1}}{\mu_{e 2}}, \\
G_{i}=\frac{P}{\left(\mu_{i} U_{0} / h_{i}^{2}\right)}, \quad b_{i}=\frac{B_{x i}}{B_{0}}, \\
K=\frac{E_{z}}{U_{0} B_{0}} \text {-loading parameter, } \\
H a_{i}=B_{0} h_{i} \sqrt{\frac{\sigma_{i}}{\mu_{i}}} \text {-Hartmann number, } \\
R m_{i}=U_{0} h_{i} \sigma_{i} \mu_{e i} \text {-magnetic Reynolds number. }
\end{gathered}
$$

With the above nondimensional quantities, the governing equations become

$$
\begin{gathered}
\frac{d^{2} u_{i}^{*}}{d y_{i}^{* 2}}-H a_{i}^{2}\left(K+u_{i}^{*} \lambda\right) \lambda+G_{i}=0, \\
\frac{d^{2} b_{i}}{d y_{i}^{* 2}}+\lambda R m_{i} \frac{d u_{i}^{*}}{d y_{i}^{*}}=0 .
\end{gathered}
$$


The nondimensional form of the boundary and interface conditions (2.12) to (2.17) becomes

$$
\begin{gathered}
u_{1}^{*}(1)=1, \quad u_{2}^{*}(-1)=0, \\
u_{1}^{*}(0)=u_{2}^{*}(0), \\
\frac{d u_{1}^{*}}{d y_{1}^{*}}=\frac{\beta}{\alpha} \frac{d u_{2}^{*}}{d y_{2}^{*}} \quad \text { for } y_{i}^{*}=0 ; i=1,2, \\
b_{1}(1)=0, \quad b_{2}(-1)=0, \\
b_{1}(0)=b_{2}(0), \\
\frac{d b_{1}}{d y_{1}^{*}}=\delta \beta \gamma \frac{d b_{2}}{d y_{2}^{*}} \quad \text { for } y_{i}^{*}=0, i=1,2 .
\end{gathered}
$$

The solutions of (3.4) with boundary and interface conditions have the following forms:

$$
\begin{gathered}
u_{i}^{*}\left(y_{i}^{*}\right)=D_{1 i} \cosh \left(\lambda H a_{i} y_{i}^{*}\right)+D_{2 i} \sinh \left(\lambda H a_{i} y_{i}^{*}\right)+F_{i} \\
b_{i}\left(y_{i}^{*}\right)=-\frac{R m_{i}}{H a_{i}}\left[D_{1 i} \sinh \left(\lambda H a_{i} y_{i}^{*}\right)+D_{2 i} \cosh \left(\lambda H a_{i} y_{i}^{*}\right)\right]+Q_{1 i} y_{i}^{*}+Q_{2 i}
\end{gathered}
$$

where

$$
\begin{gathered}
F_{i}=\frac{G_{i}}{\lambda^{2} H a_{i}^{2}}-\frac{K}{\lambda}, \\
D_{11}=\frac{\left(1-F_{1}\right) H \sinh \left(\lambda H a_{2}\right)}{W}-\frac{L \sinh \left(\lambda H a_{1}\right)}{W}, \\
L=F_{2}+S \cosh \left(\lambda H a_{2}\right), \\
W=H \cosh \left(\lambda H a_{1}\right) \sinh \left(\lambda H a_{2}\right)+\cosh \left(\lambda H a_{2}\right) \sinh \left(\lambda H a_{1}\right), \\
H=\frac{\alpha H a_{1}}{\beta} \frac{1}{H a_{2}}, \\
S=\frac{1}{\lambda^{2}}\left(\frac{G_{1}}{H a_{1}^{2}}-\frac{G_{2}}{H a_{2}^{2}}\right), \\
D_{21}=\frac{\left(1-F_{1}\right) \cosh \left(\lambda H a_{2}\right)}{W}+\frac{L \cosh \left(\lambda H a_{1}\right)}{W}, \\
D_{12}=S+D_{11},
\end{gathered}
$$




$$
\begin{gathered}
D_{22}=H D_{21}, \\
Q_{11}=R m_{1} \lambda D_{11}+\delta \beta \gamma\left(Q_{12}-\lambda R m_{2} D_{12}\right), \\
Q_{21}=\frac{R m_{1}}{H a_{1}}\left[D_{11} \sinh \left(\lambda H a_{1}\right)+D_{21} \cosh \left(\lambda H a_{1}\right)\right]-Q_{11}, \\
Q_{12}=\frac{M_{1}+M_{2}}{1+\delta \beta \gamma}, \\
M_{1}=\frac{R m_{1}}{H a_{1}}\left\{D_{11}\left[\sinh \left(\lambda H a_{1}\right)-\lambda H a_{1}\right]+D_{21}\left[\cosh \left(\lambda H a_{1}\right)-1\right]\right\}, \\
M_{2}=\frac{R m_{2}}{H a_{2}}\left\{D_{12}\left[\sinh \left(\lambda H a_{2}\right)+\lambda \delta \beta \gamma H a_{2}\right]+D_{22}\left[1-\cosh \left(\lambda H a_{2}\right)\right]\right\}, \\
Q_{22}=\frac{R m_{2}}{H a_{2}}\left[D_{22} \cosh \left(\lambda H a_{2}\right)-D_{12} \sinh \left(\lambda H a_{2}\right)\right]+Q_{12} .
\end{gathered}
$$

\section{Temperature Distribution}

Once the velocity distributions were known, the temperature distributions for the two regions have been determined by solving the energy equation subject to the appropriate boundary and interface conditions (2.18)-(2.20). In the present problem, it has been assumed that the two walls have been maintained at constant temperatures. The term involving $\partial T / \partial x=0$ in the energy equation (2.10) drops out for such a condition. The governing equation for the temperatures $T_{1}$ and $T_{2}$ in region I and II is then given by

$$
k_{i} \frac{d^{2} T_{i}}{d y^{2}}+\mu_{i}\left(\frac{d u_{i}}{d y}\right)^{2}+\sigma_{i}\left(E_{z}+u_{i} B_{0} \lambda\right)^{2}=0
$$

In order to nondimensionalize previous equation, the following transformations have been used beside the already introduced (3.3):

$$
\Theta_{i}=\frac{T_{i}-T_{w 2}}{T_{w 1}-T_{w 2}}, \quad \xi=\frac{k_{1}}{k_{2}}
$$

With the above, nondimensional quantities (4.1) for regions I and II becomes:

$$
\frac{d^{2} \Theta_{i}}{d y_{i}^{* 2}}+\operatorname{Pr}_{i} \mathrm{Ec}_{i}\left(\frac{d u_{i}^{*}}{d y_{i}^{*}}\right)^{2}+H a_{i}^{2} \operatorname{Pr}_{i} \operatorname{Ec}_{i}\left(K+u_{i}^{*} \lambda\right)^{2}=0
$$

where

$$
\operatorname{Pr}_{i}=\frac{\mu_{i} c_{p i}}{k_{i}}, \quad \mathrm{Ec}_{i}=\frac{U_{0}^{2}}{c_{p i}\left(T_{w 1}-T_{w 2}\right)}
$$


In the nondimensional form, the boundary conditions for temperature and heat flux at the interface $y=0$ becomes

$$
\begin{gathered}
\Theta_{1}(1)=1, \quad \Theta_{2}(-1)=0, \\
\Theta_{1}(0)=\Theta_{2}(0), \\
\left.\frac{d \Theta_{1}}{d y_{1}^{*}}\right|_{0}=\left.\frac{\beta}{\xi} \frac{d \Theta_{2}}{d y_{2}^{*}}\right|_{0}, \quad y_{i}^{*}=0 .
\end{gathered}
$$

The solution of (4.3) with boundary and interface conditions has the following form:

$$
\begin{aligned}
\Theta_{i}\left(y_{i}^{*}\right)=-\frac{\operatorname{Pr}_{i} \mathrm{Ec}_{i}}{4 \lambda}\{ & \lambda\left(D_{1 i}^{2}+D_{2 i}^{2}\right) \cosh \left(2 \lambda H a_{i} y_{i}^{*}\right)+8 D_{2 i} C_{i} \sinh \left(\lambda H a_{i} y_{i}^{*}\right) \\
& +2 D_{1 i} D_{2 i} \lambda \sinh \left(2 \lambda H a_{i} y_{i}^{*}\right)+8 D_{1 i} C_{i} \cosh \left(\lambda H a_{i} y_{i}^{*}\right) \\
& \left.-2 \lambda\left(2 D_{3 i}+2 D_{4 i} y_{i}^{*}-H a_{i}^{2} C_{i}^{2} y_{i}^{* 2}\right)\right\},
\end{aligned}
$$

where

$$
\begin{gathered}
C_{i}=K+\lambda F_{i}=\frac{G_{i}}{\lambda H a_{i}^{2}}, \quad i=1,2, \\
D_{31}=\frac{1}{4 \lambda} \Im_{1}-D_{41}, \\
D_{41}=\frac{1}{4 \lambda(1+(\beta / \xi))}\left(\frac{\beta}{\xi} \Im_{1}-N^{*} \Im_{2}-\frac{\beta}{\xi} \Im_{3}+\Im_{4}\right), \\
D_{32}=\frac{1}{4 \lambda N}\left(\Im_{1}-\Im_{3}\right)-\frac{1}{N} D_{41}, \\
D_{42}=\frac{D_{41}}{N^{*}}-\frac{\Im_{4}}{4 \lambda N^{*}}, \\
\Im_{1}=\lambda\left(D_{11}^{2}+D_{21}^{2}\right) \cosh \left(2 \lambda H a_{1}\right)+8 D_{21} C_{1} \sinh \left(\lambda H a_{1}\right) \\
+2 D_{11} D_{21} \lambda \sinh \left(2 \lambda H a_{1}\right)+8 D_{11} C_{1} \cosh \left(\lambda H a_{1}\right)+2 \lambda H a_{1}^{2} C_{1}^{2}+\frac{4 \lambda}{\operatorname{Pr}_{1} \mathrm{Ec}_{1}}, \\
\Im_{2}=\lambda\left(D_{12}^{2}+D_{22}^{2}\right) \cosh \left(2 \lambda H a_{2}\right)-8 D_{22} C_{2} \sinh \left(\lambda H a_{2}\right) \\
-2 D_{12} D_{22} \lambda \sinh \left(2 \lambda H a_{2}\right)+8 D_{12} C_{2} \cosh \left(\lambda H a_{2}\right)+2 \lambda H a_{2}^{2} C_{2}^{2}, \\
\Im_{3}=\lambda\left(D_{11}^{2}+D_{21}^{2}\right)-\lambda N\left(D_{12}^{2}+D_{22}^{2}\right)+8 D_{11} C_{1}-8 D_{12} C_{2} N, \\
\Im_{4}=8 \lambda H a_{1} D_{21} C_{1}+4 \lambda^{2} D_{11} D_{21} H a_{1}-8 \lambda H a_{2} D_{22} C_{2} N^{*}-4 \lambda^{2} D_{12} D_{22} H a_{2} N^{*}, \\
N=\frac{\operatorname{Pr}_{2} E c_{2}}{\operatorname{Pr}_{1} \mathrm{Ec}_{1}}, \quad N^{*}=\frac{\beta}{\xi} N .
\end{gathered}
$$




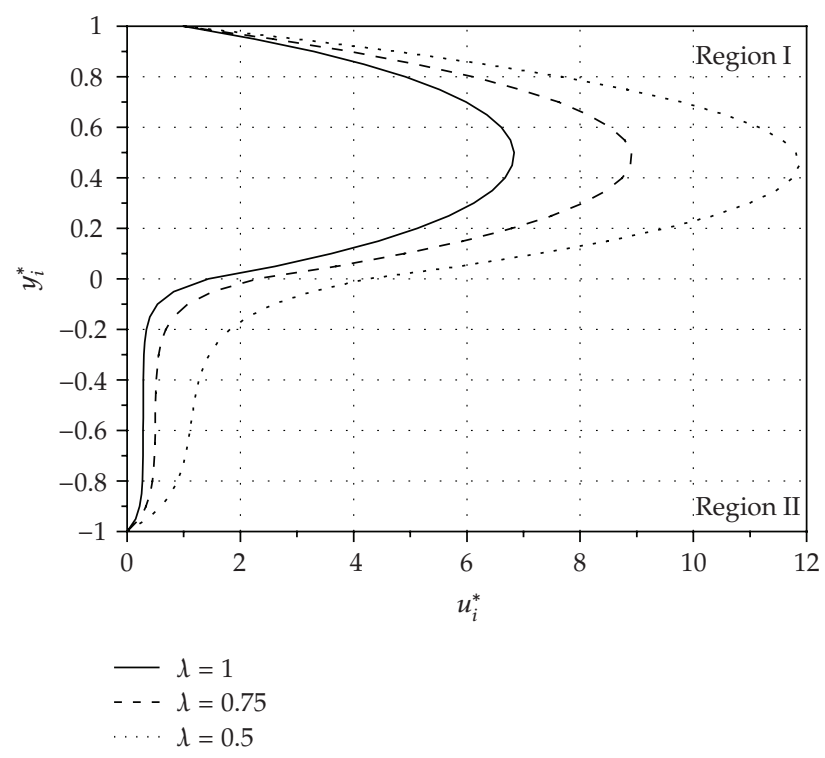

Figure 2: Velocity profiles for different values of inclination angle $\left(H a_{1}=3 ; H a_{2}=15 ; K=0\right)$.

\section{Results and Discussion}

Recent technological trends show that the use of external fields to generate the flow inside channels, such as electrohydrodynamic, MHD, and electrokinetic flows, can be more advantageous in many microscale applications. In order to show the results of the considered MHD Couette flow problem graphically, two fluids important for technical practice (selected for the development of MHD pump under the project TR35016) have been chosen, and the parameters $\alpha, \xi$ and $\gamma$ take the values of $0.677 ; 0.0647$ and 0.025 , respectively. Fluids Prandtl number is $\operatorname{Pr}_{1}=7.43$ and $\operatorname{Pr}_{2}=0.25$, while Eckert number is equal to $\mathrm{Ec}_{1}=0.0017$ and $\mathrm{Ec}_{2}=0.005$ for all the results given in Figures 2 to 13 and except in Figure 14 where it takes different values. The part of obtained results has been presented graphically in Figures 2 to 13 to elucidate the significant features of the hydrodynamic and thermal state of the flow.

Figures 2 to 4 show the effect of the magnetic field inclination angle on the distribution of velocity, temperature, and the ratio of the applied and induced magnetic field.

Figure 2 shows the effect of the angle of inclination on velocity which predicts that the velocity increases as the inclination angle increases. These results are expected because the application of a transverse magnetic field normal to the flow direction has a tendency to create a drag-like Lorentz force which has a decreasing effect on the flow velocity. Dimensionless temperature in function of angle of inclination of applied magnetic field is shown in Figure 3. In region II containing higher electrical conductivity fluid, the viscous heating is less pronounced and the influence of applied magnetic field is more expressed.

It can be seen from Figures 2 and 3 that the magnetic field flattens out the velocity and temperature profiles and reduces the flow energy transformation as the inclination angle decreases.

Figure 4 shows that the ratio of an induced and externally imposed magnetic field increases as the inclination angle of an applied field increases, for negative values of $y_{i}^{*}$. This ratio has tendency to change the sign while $\lambda$ decreases and $y_{i}^{*}$ have positive values. 


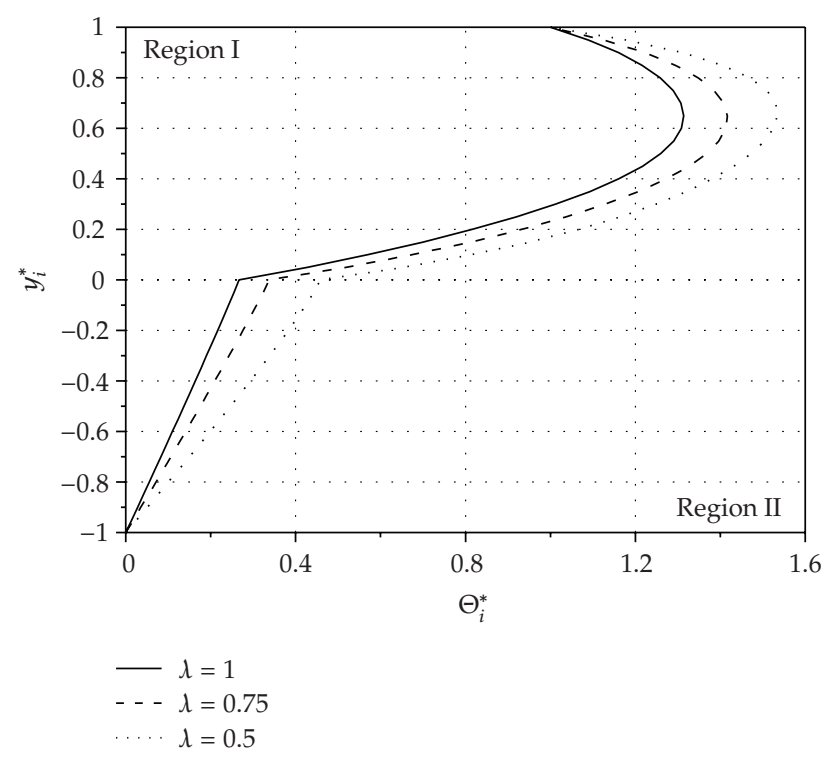

Figure 3: Temperature profiles for different values of inclination angle $\left(H a_{1}=3 ; H a_{2}=15 ; K=0\right)$.

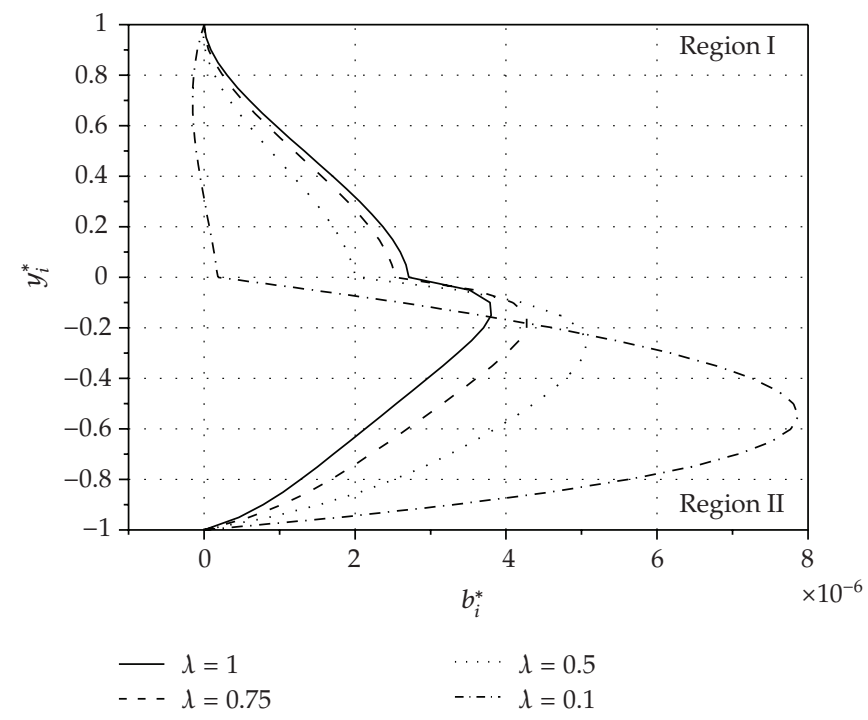

Figure 4: Ratio of an induced and externally imposed magnetic field $\left(H a_{1}=3 ; H a_{2}=15 ; K=0\right)$.

Figures 5 to 7 depict the effect of the Hartmann number, while the electric loading factor $K$ is equal to zero (so-called short-circuited case). The influence of the Hartmann number on the velocity profiles was more pronounced in the channel region II containing the fluid with greater electrical conductivity. Figure 5 illustrates the effect of the Hartmann number on the velocity field. It was found that for large values of Hartmann number, flow can be almost completely stopped in the region II, while in region I velocity decrease is significant.

The effect of increasing the Hartmann number on temperature profiles (Figure 6) in both of the parallel-plate channel regions was in equalizing the fluid temperatures. 


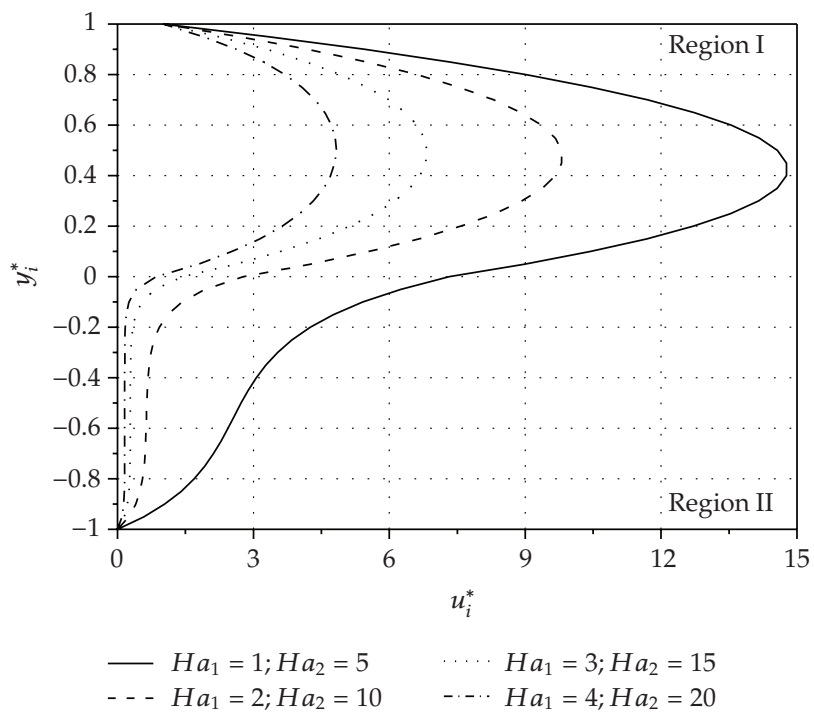

Figure 5: Velocity profiles for different values of Hartmann numbers $\mathrm{Ha}_{i}(\lambda=1)$.

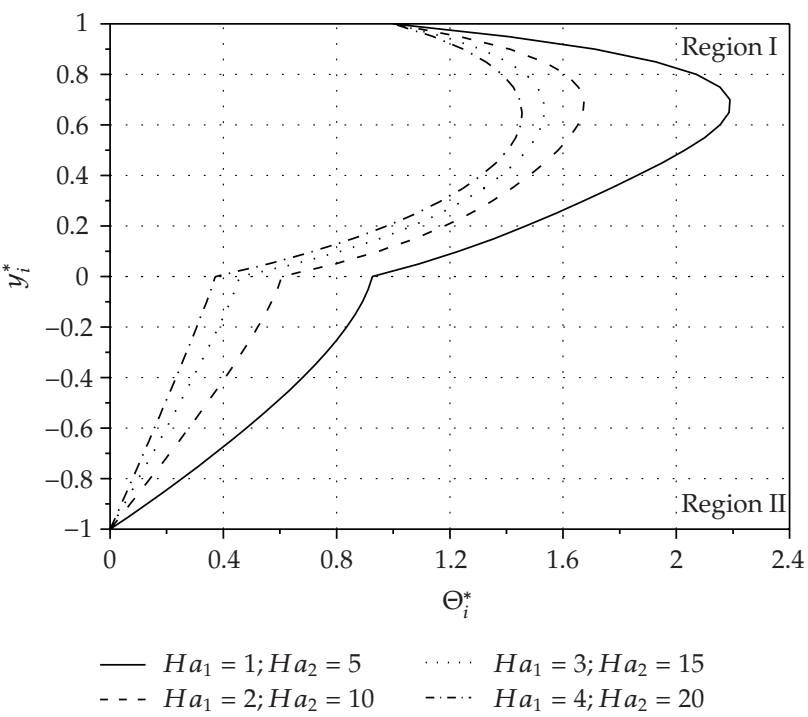

Figure 6: Temperature profiles for different values of Hartmann numbers $H a_{i}(\lambda=1)$.

The influence of the Hartmann number had quite similar effect on the ratio of induced and externally applied magnetic field as shown in Figure 7.

The influence of the induced magnetic field in the considered case is not so important, but in similar flow problems where the transversal component velocity is present, the knowledge of the imposed and induced field ratio can have great significance.

Of particular significance is the analysis when the loading factor $K$ is different from zero (value of loading factor $K$ defines the system as generator, flowmeter, or pump). Figure 8 illustrates that with the increase in the absolute value of loading factor $K$ the temperature in 


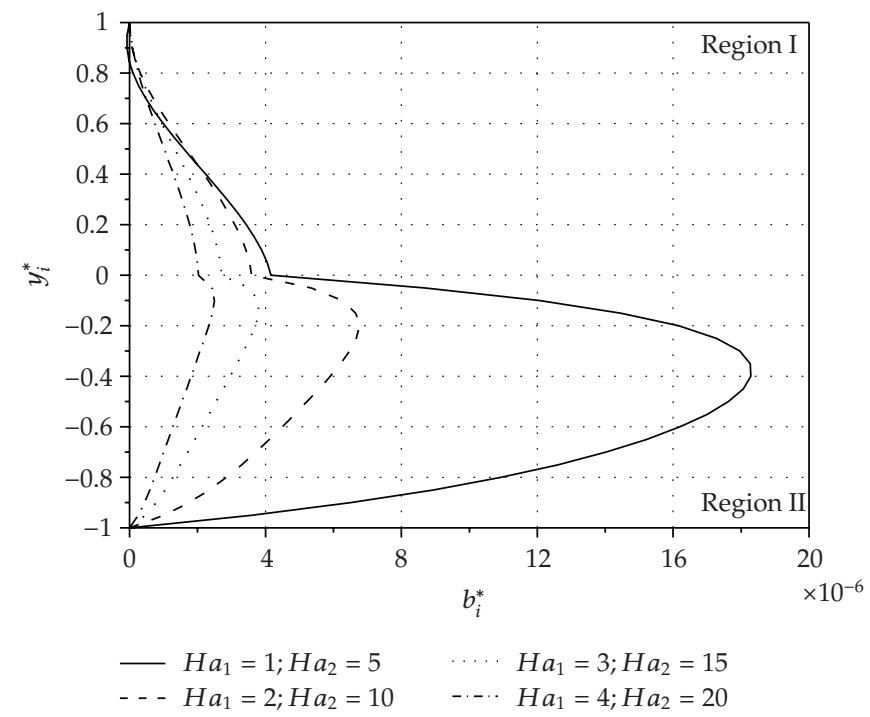

Figure 7: Ratio of an induced and externally imposed magnetic field for different values of Hartmann numbers $H a_{i}(\lambda=1)$.

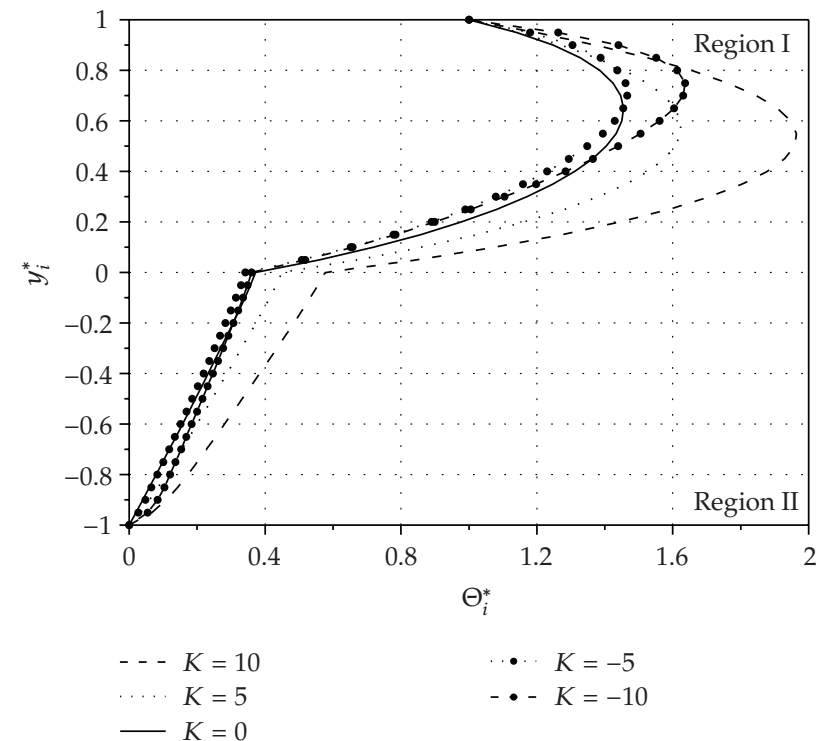

Figure 8: Temperature profiles for different values of loading factor $\left(H a_{1}=2 ; H a_{2}=10 ; \lambda=0.75\right)$.

both regions increases. In region I, viscous heating decreases while Joule heating increases, and, in region II, viscous heating increases near the lower plate and towards the middle of the channel Joule heating is more pronounced.

Figure 9 shows the effect of the loading factor on velocity, which predicts the possibility to change the flow direction. For negative $K$ values, the flow rate increases. 


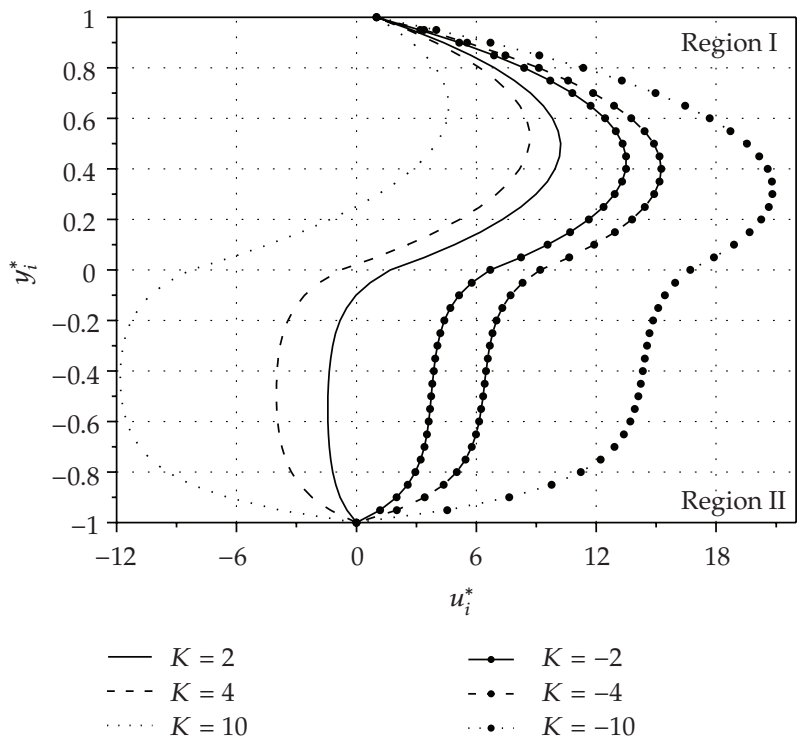

Figure 9: Velocity profiles for different values of loading factor $\left(H a_{1}=2 ; H a_{2}=10 ; \lambda=0.75\right)$.

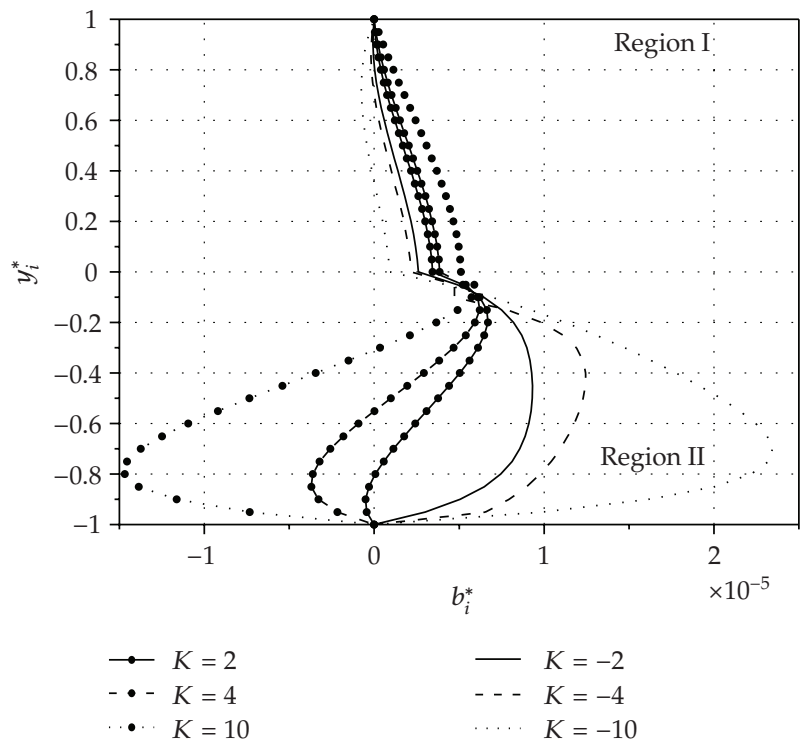

Figure 10: Ratio of an induced and externally imposed magnetic field for different values of loading factor $\left(H a_{1}=2 ; H a_{2}=10 ; \lambda=0.75\right)$.

The obtained results show that different values of the inclination angle, the Hartmann number, and the loading factor are a convenient control method for heat and mass transfer processes.

The ratio of an induced and externally imposed magnetic field had a considerable change when the loading parameter was different from zero, especially in region II. 


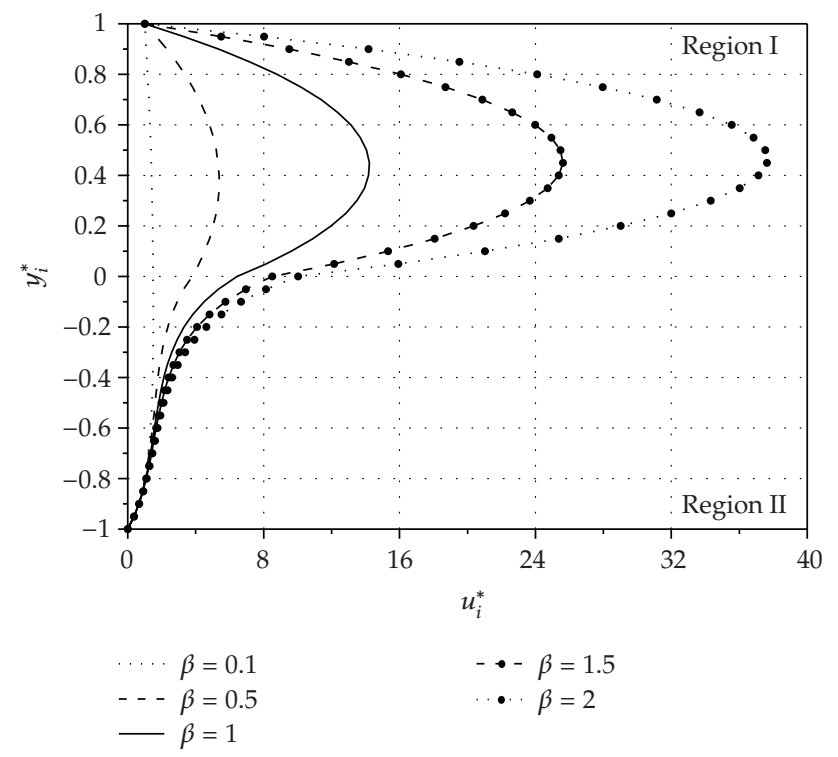

Figure 11: Velocity profiles for different values of height ratio $\beta\left(H a_{1}=1 ; H a_{2}=5 ; \lambda=1\right)$.

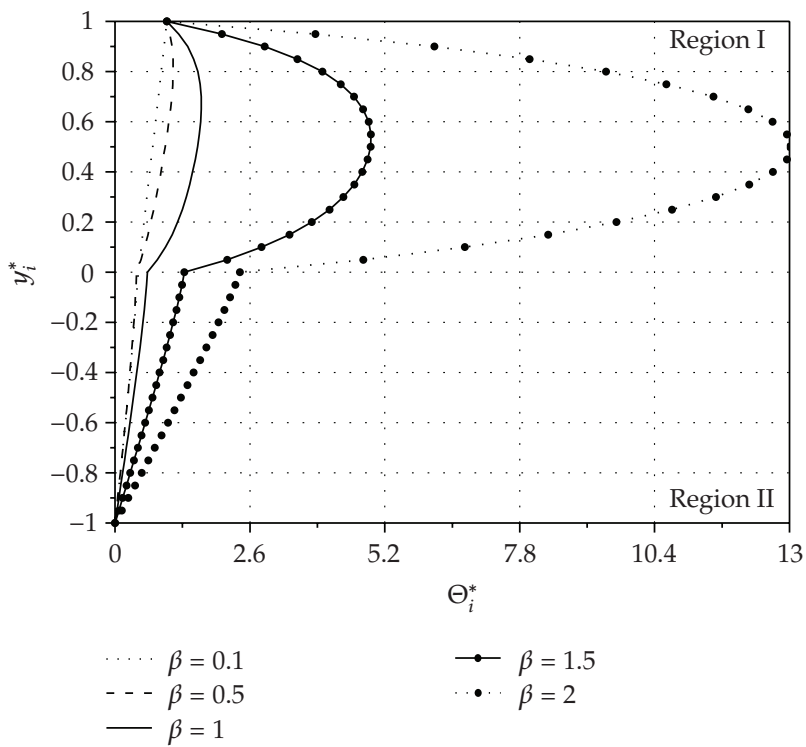

Figure 12: Temperature profiles for different values of height ratio $\beta\left(H a_{1}=1 ; H a_{2}=5 ; \lambda=1\right)$.

Figure 10 also shows a direction change of the induced field in some areas of regions I and II. This property can be used together with the change of parameters $\lambda, H a_{i}$, and $K$ in order to obtain a precise flow and heat transfer process control.

The effect of the ratio of heights of the two regions on the velocity field is shown in Figure 11. It is interesting to note that decreasing of $\beta$ flattens out velocity profiles and for small values, even change curves shape. 


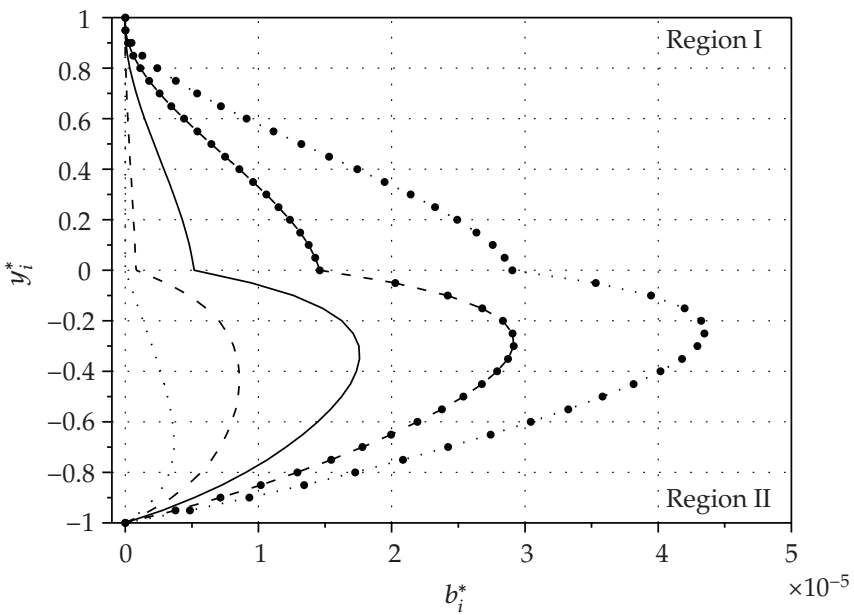

$$
\begin{aligned}
\cdots \beta & =0.1 & \cdots-\beta & =1.5 \\
-\beta & =0.5 & \cdots & \beta
\end{aligned}
$$

Figure 13: Ratio of an induced and externally imposed magnetic field for different values of height ratio $\beta\left(H a_{1}=1 ; H a_{2}=5 ; \lambda=1\right)$.

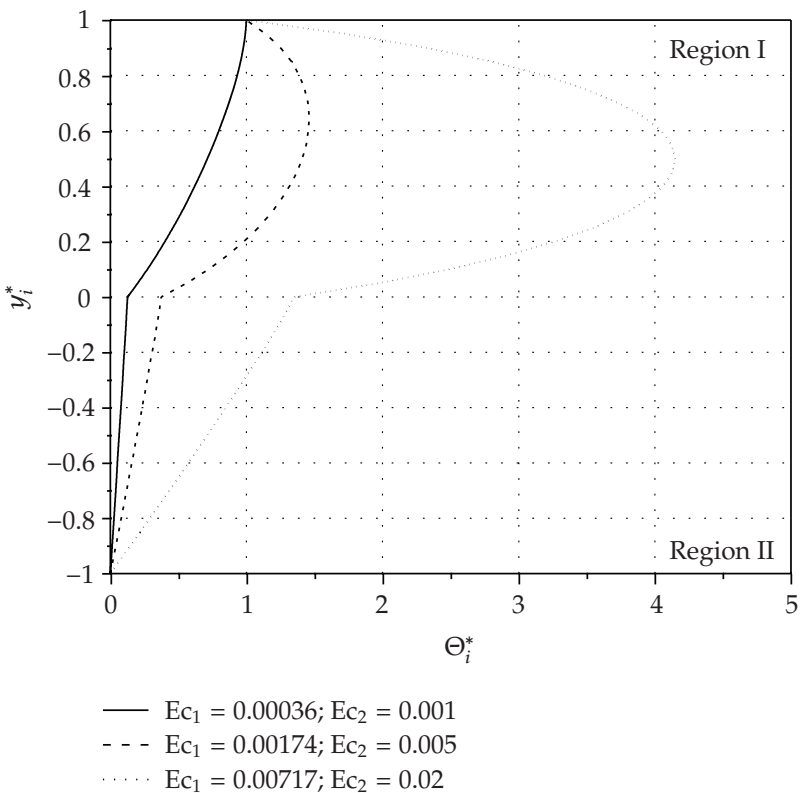

Figure 14: Temperature profiles for different values of Eckert number $\left(H a_{1}=2 ; H a_{2}=10 ; \lambda=1\right)$.

The effect of ratio of the heights of the two regions on temperature field is same as its effect on velocity field, which is evident from Figure 12. It is found that the effect of decreasing $\beta$ is to decrease the temperature field. It is also interesting to note that for small $\beta$, the ratio of induced and externally imposed magnetic field become negligible small. 
Figure 14 demonstrates the temperature distribution for different values of Eckert number Ec. It is observed that increasing values of Eckert number is to increase the temperature distribution in the flow region. Increase in Eckert number enhances the temperature because the heat energy is stored in the liquid due to the frictional heating. Temperature change is more pronounced in the region 1, while in region 2 a linear change is observed.

\section{Conclusion}

The problem of MHD Couette flow and heat transfer of two immiscible fluids in a horizontal parallel-plate channel in the presence of applied electric and inclined magnetic fields was investigated analytically. Both fluids were assumed to be Newtonian, electrically conducting, and have constant physical properties. Separate closed form solutions for velocity, temperature, and magnetic induction of each fluid were obtained taking into consideration suitable interface matching conditions and boundary conditions. The results were numerically evaluated and presented graphically for two fluids important for technical practice. Only part of the results are presented for various values of the magnetic field inclination angle, Hartmann number, loading parameter, and ratio of fluid heights in region I and II.

Furthermore, it was concluded that the flow and heat transfer aspects of two immiscible fluids in a horizontal channel with insulating walls can be controlled by considering different fluids having different viscosities and conductivities and also by varying the heights of regions. The obtained results show also that different values of the inclination angle, the Hartmann number, and the loading factor are a convenient control method for heat and mass transfer processes.

\section{Acknowledgments}

This paper is supported by the Serbian Ministry of Sciences and Technological development (Project no. TR 35016; Research of MHD flow in the channels, around the bodies and application in the development of the MHD pump). The authors wish to thank the reviewer for his careful, unbiased, and constructive suggestions that significantly improved the quality of this paper.

\section{References}

[1] E. L. Blum, M. V. Zaks, U. I. Ivanov, and Y. A. Mikhailov, Heat Exchange and Mass Exchange in Magnetic Field. Zinatne, Riga 223, 1967.

[2] K. R. Cramer and S. I. Pai, Magnetofluid Dynamics for Engineers and Applied Physicists, McGraw-Hill, New York, NY, USA, 1973.

[3] I. Tani, "Steady flow of conducting fluids in channels under transverse magnetic fields, with consideration of Hall effect," Journal of Aerospace Science, vol. 29, pp. 287-396, 1962.

[4] V. M. Soundalgekar, N. V. Vighnesam, and H. S. Takhar, "Hall and ion slip effects in MHD Couette flow with heat transfer," IEEE Transactions on Plasma Science, vol. PS-7, no. 3, pp. 178-182, 1979.

[5] V. M. Soundalgekar and A. G. Uplekar, "Hall effects in MHD Couette flow with heat transfer," IEEE Transactions on Plasma Science, vol. PS-14, no. 4, pp. 579-583, 1986.

[6] H. A. Attia, "Hall current effects on the velocity and temperature fields of an unsteady Hartmann flow," Canadian Journal of Physics, vol. 76, no. 9, pp. 739-746, 1998. 
[7] H. K. Yang and C. P. Yu, "Combined forced and free convection MHD channel flow in entrance region," International Journal of Heat and Mass Transfer, vol. 17, no. 6, pp. 681-691, 1974.

[8] G. Bodosa and A. K. Borkakati, "MHD Couette flow with heat transfer between two horizontal plates in the presence of a uniform transverse magnetic field," Journal Of Theoretical And Applied Mechanics, vol. 30, no. 1, pp. 1-9, 2003.

[9] H. A. Attia and N. A. Kotb, "MHD flow between two parallel plates with heat transfer," Acta Mechanica, vol. 117, p. 215, 1996.

[10] H. A. Attia, "Transient MHD flow and heat transfer between two parallel plates with temperature dependent viscosity," Mechanics Research Communications, vol. 26, no. 1, pp. 115-121, 1999.

[11] S. K. Ghosh, "A note on steady and unsteady hydromagnetic flow in a rotating channel in the presence of inclined magnetic field," International Journal of Engineering Science, vol. 29, no. 8, pp. 1013-1016, 1991.

[12] A. K. Borkakati and S. Chakrabarty, "Unsteady free convection MHD flow between two heated vertical parallel plates in induced magnetic field," Indian Journal of Theoretical Physics, vol. 47, pp. 143-160, 1999.

[13] O. Aydin and M. Avci, "Laminar forced convection with viscous dissipation in a Couette-Poiseuille flow between parallel plates," Applied Energy, vol. 83, no. 8, pp. 856-867, 2006.

[14] K. G. Singha, "Analytical solution to the problem of MHD free convective flow of an electrically conducting fluid between two heated parallel plates in the presence of an induced magnetic field," International Journal of Applied Mathematics and Computation, vol. 1, no. 4, pp. 183-193, 2009.

[15] R. Shail, "On laminar two-phase flows in magnetohydrodynamics," International Journal of Engineering Science, vol. 11, no. 10, pp. 1103-1108, 1973.

[16] J. Lohrasbi and V. Sahai, "Magnetohydrodynamic heat transfer in two-phase flow between parallel plates," Applied Scientific Research, vol. 45, no. 1, pp. 53-66, 1988.

[17] S. Alireza and V. Sahai, "Heat transfer in developing magnetohydrodynamic Poiseuille flow and variable transport properties," International Journal of Heat and Mass Transfer, vol. 33, no. 8, pp. 17111720, 1990.

[18] M. S. Malashetty, J. C. Umavathi, and J. P. Kumar, "Convective magnetohydrodynamic two fluid flow and heat transfer in an inclined channel," Heat and Mass Transfer, vol. 37, no. 2-3, pp. 259-264, 2001.

[19] M. S. Malashetty, J. C. Umavathi, and J. P. Kumar, "Two fluid flow and heat transfer in an inclined channel containing porous and fluid layer," Heat and Mass Transfer, vol. 40, no. 11, pp. 871-876, 2004.

[20] J. C. Umavathi, A. Mateen, A. J. Chamkha, and A. A. Mudhaf, "Oscillatory Hartmann two-fluid flow and heat transfer in a horizontal channel," International Journal of Applied Mechanics and Engineering, vol. 11, no. 1, pp. 155-178, 2006.

[21] J. C. Umavathi, A. J. Chamkha, A. Mateen, and A. Al-Mudhaf, "Unsteady two-fluid flow and heat transfer in a horizontal channel," Heat and Mass Transfer, vol. 42, no. 2, pp. 81-90, 2005.

[22] J. C. Umavathi, I. C. Liu, and J. Prathap Kumar, "Magnetohydrodynamic Poiseuille-Couette flow and heat transfer in an inclined channel," Journal of Mechanics, vol. 26, no. 4, pp. 525-532, 2010.

[23] H. B. Haim, Z. Jianzhong, Q. Shizhi, and X. Yu, "A magneto-hydrodynamically controlled fluidic network," Sensors and Actuators B, vol. 88, no. 2, pp. 205-216, 2003.

[24] S. K. Hussameddine, J. M. Martin, and W. J. Sang, "Analytical prediction of flow field in magnetohydrodynamic-based microfluidic devices," Journal of Fluids Engineering, vol. 130, no. 9, p. 6, 2008 .

[25] M. Yi, S. Qian, and H. Bau, "A magnetohydrodynamic chaotic stirrer," Journal of Fluid Mechanics, vol. 468, pp. 153-177, 2002.

[26] M. C. Weston, M. D. Gerner, and I. Fritsch, "Magnetic fields for fluid motion," Analytical Chemistry, vol. 82, no. 9, pp. 3411-3418, 2010.

[27] S. K. Ghosh, "Effects of Hall current on MHD Couette flow in a rotating system with arbitrary magnetic field," Czechoslovak Journal of Physics, vol. 52, no. 1, pp. 51-63, 2002.

[28] G. S. Seth, R. Nandkeolyar, N. Mahto, and S. K. Singh, "MHD couette flow in a rotating system in the presence of an inclined magnetic field," Applied Mathematical Sciences, vol. 3, no. 57-60, pp. 2919-2932, 2009.

[29] S. Smolentsev, S. Cuevas, and A. Beltrán, "Induced electric current-based formulation in computations of low magnetic Reynolds number magnetohydrodynamic flows," Journal of Computational Physics, vol. 229, no. 5, pp. 1558-1572, 2010.

[30] P. J. Bhuyan and K. S. Goswami, "Effect of magnetic field on MHD pressure drop inside a rectangular conducting duct," IEEE Transactions on Plasma Science, vol. 36, no. 4, pp. 1955-1959, 2008. 


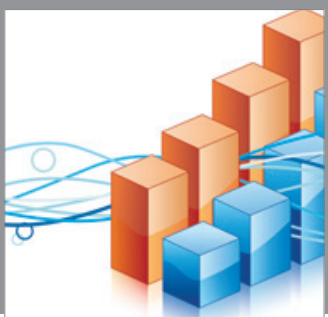

Advances in

Operations Research

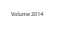

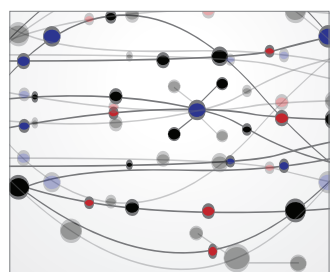

\section{The Scientific} World Journal
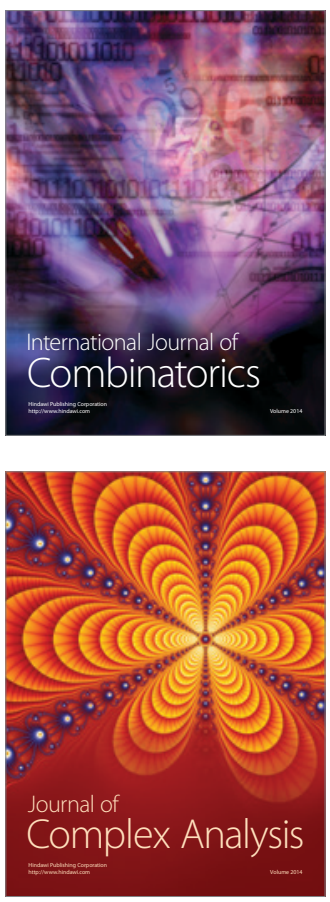

International Journal of

Mathematics and

Mathematical

Sciences
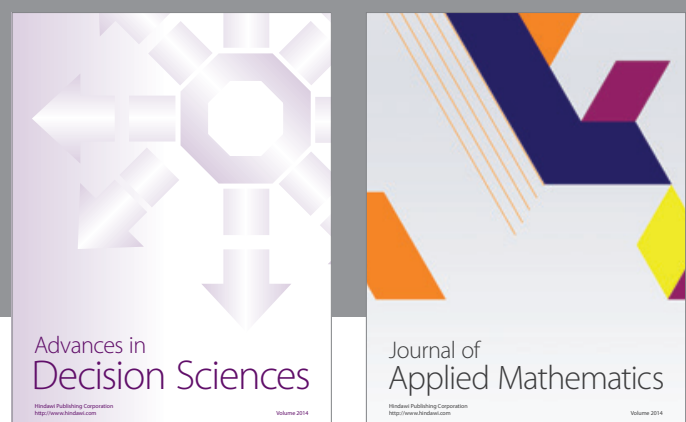

Journal of

Applied Mathematics
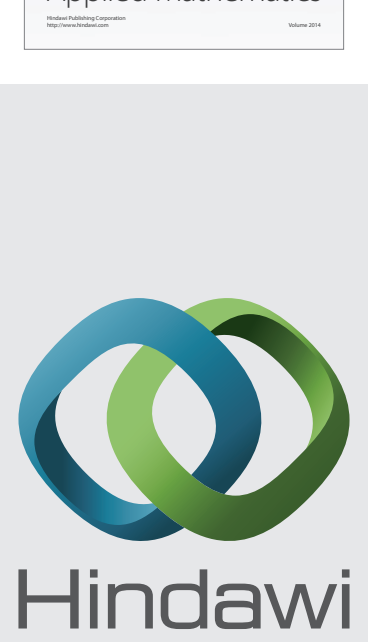

Submit your manuscripts at http://www.hindawi.com
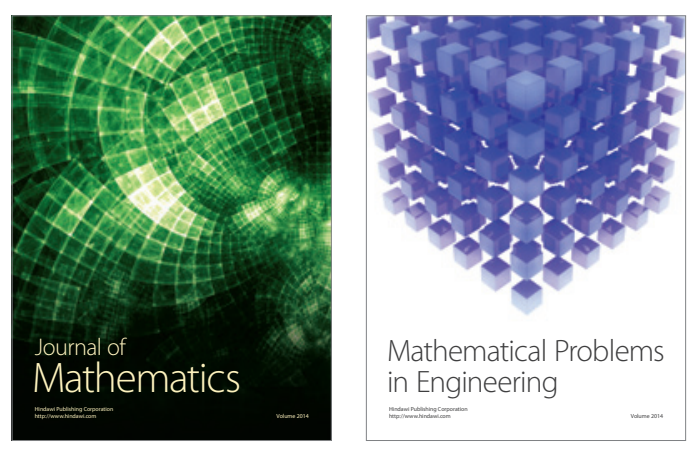

Mathematical Problems in Engineering
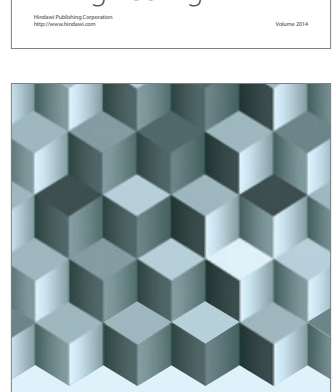

Journal of

Function Spaces
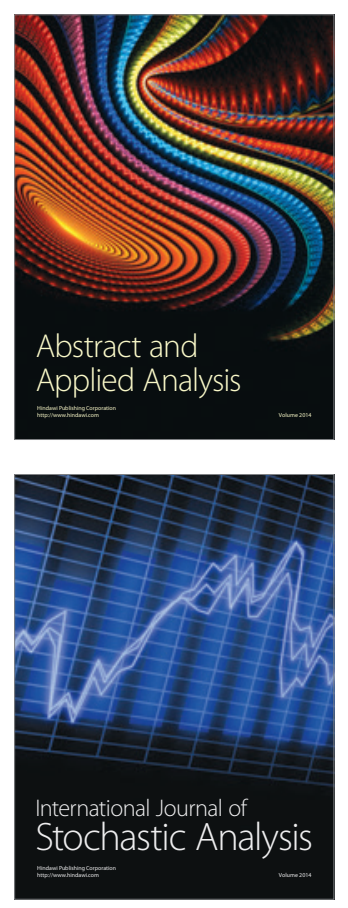

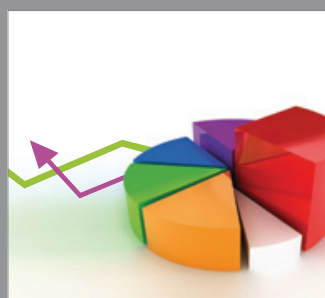

ournal of

Probability and Statistics

Promensencen
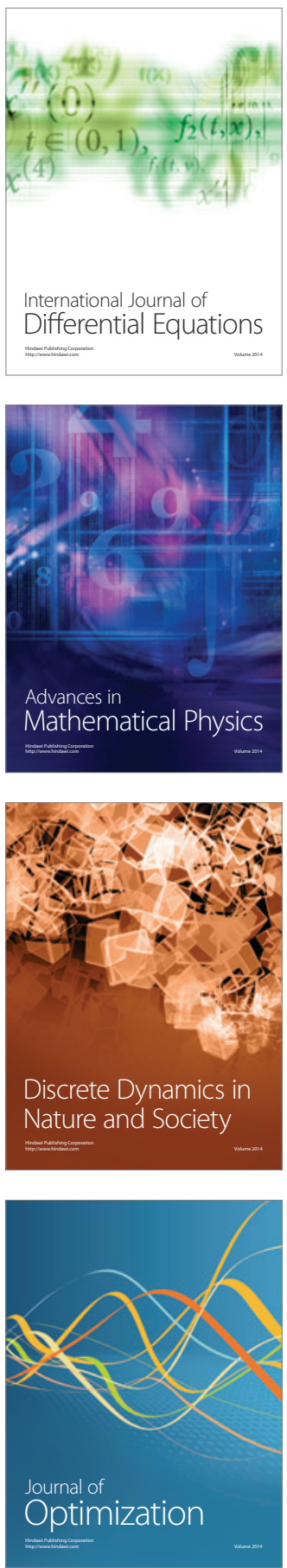